\title{
Role of intravenous paracetamol as an adjunct to epidural analgesia in immediate postoperative period in abdominal cancer surgeries
}

\author{
Navnath K. Sonale ${ }^{1}$, Devanand B. Pawar ${ }^{2, *}$ \\ 1,2 Assistant Professor, Dept. of Anaesthesia, Swami Ramanand Teerth Rural Medical College, Ambajogai, Maharashtra, India
}

*Corresponding Author:

Email: drpawardb@gmail.com

Accepted: $09^{\text {th }}$ August, 2017

\begin{abstract}
Aims and Objectives: To evaluate analgesic efficacy and side effects of intravenous paracetamol, also assess IV-PCA morphine consumption in patients undertaken abdominal cancer surgery.

Materials and Methods: Total 160 ASA grade I and II patients of both the sex, between age 18-70 years were randomly divided in two equal groups to receive either IV paracetamol $1 \mathrm{gm}$ or no paracetamol after surgery. Hemodynamic parameters, pain intensity scores, amount of morphine consumption and side effects were recorded.

Results: The control group had higher mean heart rate, mean arterial blood pressures, mean respiratory rate than paracetamol group. Pain intensity score was much higher in control group at any time which needed considerably higher dose of rescue analgesic (IV morphine) for pain control than paracetamol group. We found no significant difference statistically in sedation scores in patients using patient controlled analgesia in either group. Side effects were found to be lower in paracetamol group compared to control group but difference was not significant statistically.

Conclusion: Paracetamol is safe and effective drug for postoperative analgesia with negligible side effects.
\end{abstract}

Keywords: IV paracetamol, IV-PCA morphine, Abdominal cancer surgery, Epidural Analgesia, Pain scores, Sedation score.

\section{Introduction}

Postoperative pain is one of the most disagreeable experiences for a patient undergoing surgery. The prevalence of postoperative pain varies individually and is related to type and site of surgery. Deliberate action should be taken to treat the pain prophylactically. If postoperative pain does develop, it should be managed early and assertively. As the postoperative severe pain not only brings a delay in discharge and poorer patient satisfaction, but also can create a hyperalgesic condition recognized as persistent postoperative pain (PPP). ${ }^{1}$ Therefore, it is in the anesthesiologist's best attention to be aware of the severity of this problem and of all the pharmacological agents used to treat and prevent postoperative pain.

The goal for postoperative pain management is to reduce or eliminate pain and discomfort with minimum side effects, in a very cost effective manner. Opioid therapy and non-steroidal anti-inflammatory drugs (NSAIDS) are recommended as a first-choice medication for postoperative pain management, but it is associated with several undesirable adverse effects. ${ }^{2}$ Therefore multimodal and preventive approach to provide postoperative analgesia is gaining popularity these days. For this reason paracetamol is now being explored for same.

Paracetamol is a nonopioid analgesic ${ }^{3}$ and thought to exert its analgesic activity by inhibiting the synthesis of prostaglandins in central nervous system. After the approval of intravenous paracetamol (acetaminophen) in 2010, it has been widely used for fever and pain in children and adults. Injectable paracetamol administration provides pain relief within 5-10 minutes. The peak analgesic effect is attained in 1 hour and the duration of this effect lasts for 4-6 hour. ${ }^{4}$ This time course can be altered, if speed of infusion is slowed down then the onset and time to peak period will be prolonged. It is devoid of any major adverse effects like respiratory and circulatory depression and has no sedative effect. Analgesic effect of paracetamol has been assumed to relate directly to it blood concentration, the bioavailability of intravenous paracetamol is $100 \%$, so it acts as potent analgesic as compared to other formulations.

The present study was carried out with an objective to evaluate the analgesic efficacy and side effects of intravenous paracetamol after abdominal cancer surgeries.

\section{Materials and Methods}

This was an open labelled randomized control study. After obtaining patient's written informed consent and hospital ethics committee approval, a total 160 adult patients of average built in the age groups between 18-70 years, ASA I and II of either sex undergoing lower abdominal cancer surgery, under general anaesthesia and epidural analgesia were included. Exclusion criteria included patients having past history of allergy to paracetamol, NSAIDs/ aspirin or any other drug used in the study, age $<18$ yrs, ASA III or more, pregnant/ breast feeding women, patients with severe organ dysfunction such as liver or hepatic failure, alcohol or drug abuse, psychiatric illness, history of recent myocardial infarction i.e. within 7 
days, inability to use numerical rating scale, serum bilirubin more than $1.8 \mathrm{mg} / \mathrm{dl}$, aspartate aminotransferase or alanine aminotransferase $>1.5$ of the upper limit of normal, inability to give informed consent, patients on antiepileptic drugs and patients on ventilatory support for more than 24 hours. After detailed pre-anaesthetic evaluation, patients were explained the 11 points $(0-10)$ numeric rating scale (NRS) to assess pain score.

Then under all aseptic precautions thoracic or lumbar epidural catheter was inserted and a test dose of lignocaine $1 \%$ with 1:200000 adrenaline $3 \mathrm{ml}$ was given and an anaesthetic band was confirmed. Patients were anaesthetised using fentanyl $2 \mathrm{mcg} / \mathrm{kg}$ and then induced with propofol $2 \mathrm{mg} / \mathrm{kg}$ IV. After confirming bag mask ventilation IV vecuronium bromide $0.1 \mathrm{mg} . \mathrm{kg}$ was given and patient was intubated and was maintained on Isoflurane/sevoflurane with $\mathrm{N} 2 \mathrm{O}: \mathrm{O} 2$ 60:40 on circle absorber system with IPPV. Intraoperatively, IV fentanyl $1 \mathrm{mcg} / \mathrm{kg}$ boluses were given as required. IV ondansetron $0.1 \mathrm{mg} . \mathrm{kg}$ was given for PONV before reversal of anaesthesia. All patients received intraoperatively inj. Bupivacaine $0.125 \%$ infusion at $5 \mathrm{ml} / \mathrm{hr}$.

The patients were randomized pre-operatively in two equal groups to receive either IV paracetamol $1 \mathrm{gm}$ (Paracetamol group) or no paracetamol (Control group) after surgery.

Control group (80 patients): received $0.125 \%$ bupivacaine epidurally via an elastomeric pump @ 5ml per hour, IV PCA (morphine) $1 \mathrm{mg} / \mathrm{bolus}, 10$ minutes lockout interval, not $>6$ doses per hour as rescue analgesic. Paracetamol group (80 patients): received study drug IV paracetamol 1 gram at 6 hours interval and $0.125 \%$ bupivacaine epidurally via an elastomeric pump @ 5ml per hour, IV-PCA (morphine) $1 \mathrm{mg} /$ bolus,
10 minutes lockout interval, not $>6$ doses per hour as rescue analgesia.

All patients received 4 doses of the study drug at 6 hr intervals (for 24 postoperative hours). The first drug dose was given after patient shifted from Operation Theater in recovery room. Observer, who monitored the patient, assessed the pain relief for 24 hours postoperatively. Patients who did not have adequate pain relief were given patient controlled IV morphine analgesia through a dedicated IV line in a dose of $1 \mathrm{mg} /$ bolus with a lockout interval of 10 minutes allowing 6 doses per hour to titrate pain relief (maximum dose $10 \mathrm{mg} /$ day).

Pain scores assessed at rest and during deep breathing at $0,1,2,6,12,18$, and 24 hours. Mean heart rate, mean arterial pressure, respiratory rate (RR), 11 points $(0-10)$ numeric rating scale (NRS) score at rest and at deep breath, PONV score, Modified Ramsay Sedation score and Side effects were recorded. Also the amount of morphine consumption and number of attempts and demand dose of morphine was recorded.

\section{Statistical Analysis}

Data analysis was done with SPSS version 18.0 software (SPSS Inc., Chicago, IL). Continuous data were presented as a median and range (min-max) and compared between groups using a Mann-Whitney test. A Pearson x 2-test was used for categorical data. NRS score was compared between groups across time intervals using a ANOVA or Mann-Whitney test (as appropriate) with Bonferroni correction for multiple comparisons: for each parameter depending on time intervals, the alpha level of each individual test was divided by the number of observation to ensure that overall type one error remains 0.05 . An analysis was conducted on an intention-to-treat basis.

\section{Observations and Results}

The demographic variables and duration of surgery were comparable in both the groups and difference was not significant statistically (Table 1).

Table 1: Demographic data and Duration of surgery

\begin{tabular}{|l|c|c|}
\hline \multicolumn{1}{|c|}{ Parameters } & Group P & Group C \\
\hline Age (yrs) & $51.63 \pm 13.02$ & $51.38 \pm 12.73$ \\
\hline Weight $(\mathrm{kg})$ & $61.75 \pm 8.13$ & $59.63 \pm 8.77$ \\
\hline Males/Females & $47 / 33$ & $35 / 45$ \\
\hline ASA status I/II & $52 / 28$ & $61 / 19$ \\
\hline Duration of surgery & $299.50 \pm 122.55$ & $270.13 \pm 120.02$ \\
\hline
\end{tabular}

The mean heart rate (HR) and mean arterial blood pressures (MAP) was higher in control group than in paracetamol group. For HR statistically significant difference of $\mathrm{P}<0.05$ was observed at $6,12,18,24$ hours and insignificant difference $(\mathrm{P}>0.05)$ at 0,1 and 2 hours whereas for MAP statistical significant difference observed at all the measuring intervals except at 0,24 hours $(\mathrm{P}>0.27)$, (Fig.1). The respiratory rates were stable in both groups; and it was found that mean respiratory rate was higher in control group compared to paracetamol group which was significant statistically $(\mathrm{P}<0.02)$. 


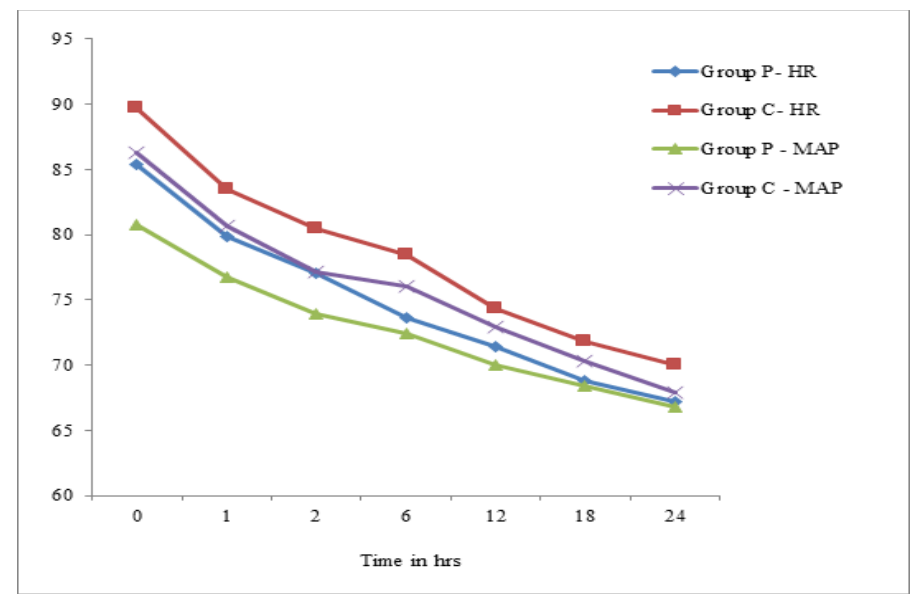

Fig. 1: Changes in Heart Rate and Mean Arterial Pressure

Table 2: Comparison of VAS score at rest and during movement between two groups

\begin{tabular}{|l|c|c|c|c|}
\hline \multirow{2}{*}{$\begin{array}{c}\text { Time } \\
\text { Interval }\end{array}$} & \multicolumn{2}{|c|}{ At Rest } & \multicolumn{2}{c|}{ During Movement } \\
\cline { 2 - 5 } & Group P & Group C & Group P & Group C \\
\hline 0min & 2.11 & 2.31 & 3.94 & 3.61 \\
\hline $1 \mathrm{hr}$ & 2.65 & 3.21 & 3.95 & 4.03 \\
\hline $2 \mathrm{hr}$ & 2.20 & 3.05 & 3.58 & 3.96 \\
\hline $6 \mathrm{hr}$ & 1.83 & 2.61 & 3.26 & 3.95 \\
\hline $12 \mathrm{hr}$ & 1.41 & 2.36 & 2.86 & 3.74 \\
\hline $18 \mathrm{hr}$ & 1.05 & 2.13 & 2.31 & 3.49 \\
\hline $24 \mathrm{hr}$ & .53 & 1.99 & 1.71 & 3.38 \\
\hline
\end{tabular}

We found statistically significant difference $(\mathrm{P}<0.01)$ in mean pain scores at rest and at movement between the groups with pain scores higher in control group at all measurement intervals but at rest, on 0 hour interval and at movement on 0 and 1 hour interval it was not significant statistically (Fig. 2).

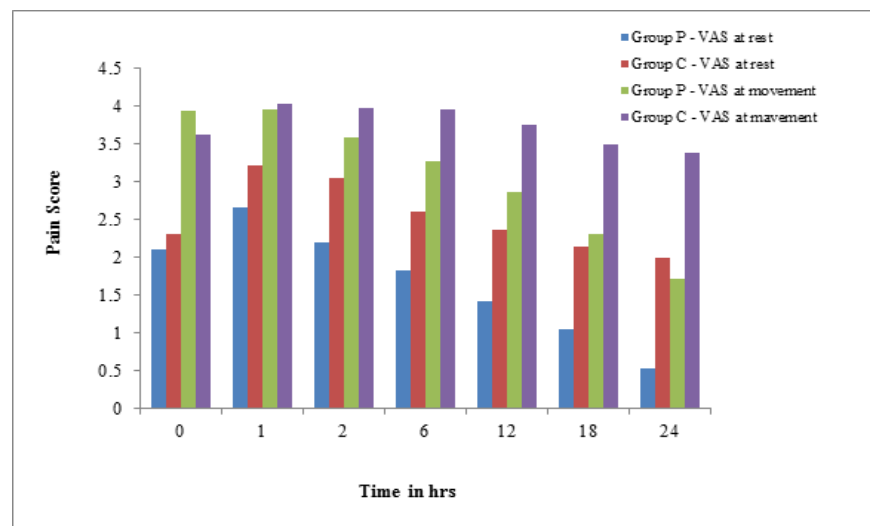

Fig. 2: Comparison of pain score (VAS) at rest and at movement

There were statistically significant difference in need for rescue analgesic (IV PCA morphine) in both the groups at all intervals except during at 1 hour interval where there was insignificance $(P=0.4)$. Total morphine consumed during 24 hours was also higher in control group $(47.75 \mathrm{mg}$ ) compared to paracetamol group (24.5mg) which was statistical significant with opioid sparing effect $51 \%$. There was no significant difference statistically ( $\mathrm{P}$ $>0.4$ ) found in sedation scores in patients using patient controlled analgesia in either group.

Incidence of nausea and vomiting was found to be lower in paracetamol group compared to control group but difference was not significant statistically. Hepatic damage, a concern with the use of IV paracetamol $1 \mathrm{gm}$, was not encountered with doses of paracetamol up to 4 grams per day. 


\section{Discussion}

The current study reveals a significant opioid sparing effect through IV paracetamol $1 \mathrm{gm}$ for postoperative pain relief after abdominal surgeries. Literature review showed worldwide up to $70 \%$ of patients receive ineffective, inadequate or delayed postoperative pain relief. ${ }^{5}$ In postoperative period, multimodal analgesia strategy is used to obtain maximum pain relief with minimum side effects. Combination of paracetamol and/or NSAIDS, opioids and local anaesthetics through regional anaesthesia are component of multimodal analgesia. Intravenous paracetamol is the water-soluble formulation, with negligible side effects; its efficacy for treating postoperative pain has been investigated in several studies. Oral route and rectal form of administration is the convenient but it is unreliable and erratic for intraoperative patients. It gives a variable peak plasma concentration reached later at 2 to 3 hours. So for the most drugs, iv administration is more reliable and reaches peak concentrations faster compared with oral routes, as proven for paracetamol. ${ }^{6}$ Paracetamol readily prepared in solution has only recently become available, so the literature is still scarce. In any case, the bioequivalence study has revealed that $1 \mathrm{gm}$ of paracetamol is bioequivalent to $2 \mathrm{gm}$ of propacetamol and is safer at the local level. ${ }^{7}$

The demographic data in terms of age, weight, ASA status, male/female ratio, duration of surgery were comparable between two groups and had no influence on outcome of the study. Haemodynamic status of patients those receiving paracetamol $1 \mathrm{gm}$ was significantly better as compared to that not receiving, where there was statistically significant increase in heart rate, blood pressure, respiratory rate. The previous studies show that the use of intravenous paracetamol had resulted in better pain control and haemodynamic stability. Our results were consistent with results of previous studies. $^{8-10}$

There was statistically significant difference $(\mathrm{P}<0.01)$ in mean pain scores at rest and during movement between the groups. Except at 0 hour interval, the pain scores at rest were higher in control group at all intervals of measurement where the difference in pain scores was not statistically significant $(P=0.17)$. The pain scores during movement were higher in the control group at all intervals of measurement except at 0 hour interval $(\mathrm{P}=0.058)$ and at 1 hour interval $(\mathrm{P}=0.55)$ where it was not statistically significant. Remy et $\mathrm{al}^{8}$ reported that adding acetaminophen to PCA was associated with a morphine-sparing effect of $20 \%$ over the first postoperative $24 \mathrm{~h}$. Moller et $\mathrm{al}^{9}$ found that, acetaminophen $1 \mathrm{gm}$ was superior to placebo regarding analgesic efficacy. Eremenko et $\mathrm{al}^{10}$ found early postoperative use of paracetamol after myocardial revascularization reduces the intake of opioids and diminishes the intensity of pain syndrome within first hours after extubation.

It was noticed that total morphine consumed during 24 hours was also higher in control group than paracetamol group which was statistical significant $(\mathrm{P}=$ $0.00)$ with $51 \%$ opioid sparing effects. This showed that those patients receiving IV paracetmol were having superior pain relief as compared to patients not receiving paracetamol that is control group. Effective and non-sedating pain relief in the postoperative period is essential to minimize the risk of delayed recovery and discharge from hospital. Use of IV PCA morphine is associated with several side effects such as sedation, nausea, vomiting, pruritus, delayed respiratory depression but incidence of these side effects with the use of intravenous paracetamol are minimal.

The side effect of nausea and vomiting was to be higher in control group as compared to paracetamol group. The sedation score and side effects in both the groups were not statistically significant.

Thus, paracetamol group had better hemodynamic stability as compared to control group. Side effects such as nausea, vomiting and sedation attributed to opioids like morphine can be decreased with the use of this opioid sparing paracetamol.

\section{Conclusion}

The observations of present study may be concluded that IV paracetamol is safe and effective drug for postoperative analgesia with negligible side effects. Since paracetamol's side effect profile is considerably superior, and an intravenous form produces rapid pain relief in those patients who are contraindicated for oral formulations and absorption of oral formulation is erratic such as after abdominal surgery are very useful when other routes are less feasible.

\section{Acknowledgement}

The authors sincerely thanks the Department of Anaesthesiology, surgery, other staff of operation theatre and administration of Tata Memorial Hospital, Mumbai, Maharashtra, for permission to study and providing facility to carry out the work.

\section{References}

1. Johansen A, Romundstad L, Nielsen CS, Schirmer H, and Stubhaug A. Persistent postsurgical pain in a general population: prevalence and predictors in the Tromso study. Pain 2012;153(7):1390-6.

2. Marret E, Kurdi O, Zufferey P, Bonnet F. Effect of nonsteroidal antiinflammatory drugs on patients controlled analgesia morphine side effects: meta-analysis of randomized controlled trials. Anesthesiology 2005;102:1249-60.

3. Bjune K, Stubhaug A, Dodgson MS, Breivik H. Additive analgesic effect of codeine and paracetamol can be detected in strong, but not moderate, pain after Caesarean section. Baseline pain-intensity is a determinant of assay- 
sensitivity in a postoperative analgesic trial. Acta

Anaesthesiology Scandinavica 1996;40:399-407.

4. Arici S, Gurbet A, Türker G, Yavascaoglu B, Sahin S.

Preemptive analgesic effects of intravenous paracetamol in total abdominal hysterectomy. Medical

Agri.2009;21:54-61.

5. Baer GA, Rorarius MG, Kolehmainen S, SelinS. The effect of paracetamol or diclofenac administered before operation on postoperative pain and behaviour after adenoidectomy in small children. Anaesthesia 1992,47:1078-80.

6. Chris $\mathrm{P}$, Daphne $\mathrm{S}$. The role of intravenous acetaminophen in acute pain management. Pain Manag Nurs 2012;13(2):107-24.

7. Garcia Rodriguez RL, Jick H. Risk of upper gastrointestinal bleeding and perforation associated with individual non-steroidal anti-inflammatory drugs. Lancet 1994;343:769-72.

8. Remy C, Marret E and Bonnet F. Effects of acetaminophen on morphine side-effects and consumption after major surgery: meta-analysis of randomized controlled trials $\mathrm{Br}$. J. Anaesth 2005;94(4):505-13.

9. Moller PL, Juhl GI, Payen-Champenois C, Skoglund LA. Intravenous acetaminophen (paracetamol): comparable analgesic efficacy, but better local safety than its prodrug, proparacetamol, for postoperative pain after third molar surgery. Anesth Analg 2005;101(1):90-6.

10. EremenkoAA, KuslievaEV. Analgesic and opioid-sparing effects of intravenous paracetamol in the early period after aortocoronary bypass surgery Anesteziol Reanimatol 2008;(5):11-4. 